\title{
Gateway cities: círculos bancarios, concentración y dispersión en el ambiente urbano brasileño***
}

\begin{abstract}
This exploratory study shows how the major banks operating in Brazil in 2003 apply the two global practices - concentration and dispersion - in the Brazilian urban environment. Four global and two domestic location strategies of those banks were identified. The strategies were found employing the multivariate method of principal component analysis in a matrix of 54 cities versus 31 banks. In the light of world city functions, the gateway city concept as defined by Alan Pred was the basis for interpreting the six bank location strategies. The hinterland of a world city was understood as its functional links to other cities in the country, created in the global economy by advanced producer services in the world city. According to the results, the city of Sao Paulo articulates the national and the global economies as the main gateway city in Brazil. Additionally, they point to Curitiba as an alternative Brazilian gateway city. The results also show that high-accumulated sales in a city contribute only slightly to the city performance in global banking circles.
\end{abstract}

Key words: banking system, location strategies, brazilian cities, world city, gateway city, world city network

\section{Resumen}

Este estudio muestra cómo los principales bancos en operación en Brasil, durante el 2003, aplican las dos prácticas de la globalización -concentración y dispersión- en el ambiente urbano brasileño. Cuatro estrategias globales de localización de estos bancos y dos domésticas fueron identificadas. Las seis estrategias resultan de la aplicación de uno de los métodos de análisis multivariado, el análisis de componentes principales, en una matriz compuesta por 54 ciudades y 31 bancos. Consideradas las funciones de la ciudad mundial, se adoptó el concepto de gateway city, de Alan Pred, para interpretar las respectivas estrategias. La hinterland de la ciudad mundial fue entendida como sus enlaces funcionales con otras ciudades del país, creados en la economía global por los productores de servicios con alto valor agregado, ubicados en la ciudad mundial. Los resultados revelan que San Pablo articula las economías nacional y global como gateway city y que Curitiba emerge como una alternativa a San Pablo en esta función. Además, muestran que la producción en ventas de importantes ciudades de la red urbana brasileña poco contribuye para el desempeño de estas urbes en el contexto globalizado de los bancos.

Palabras clave: sistema bancario, estrategias de localización, ciudades brasileñas, ciudad mundial, gateway city, red de ciudades mundiales. 


\section{Introducción}

$\mathrm{L}$ as políticas nacionales de regulación y de inserción global, respectivamente, explicitan tanto el ingreso y la presencia de los productores globales de servicio como las alternativas de inserción global de los diferentes países. En el ámbito de las opciones de inserción global, América Latina se muestra dependiente de las demandas de la globalización de Asia y de las economías centrales. Confirmando las características del proceso de globalización, la producción destinada a los mercados internacionales ocurrió lejos de los centros tradicionales. Así, ciudades secundarias se hacen relevantes para la red urbana nacional y para las relaciones económicas internacionales. Aunque existan cambios en la estructura productiva de las ciudades involucradas, las fuerzas centrífugas no superaron las centrípetas, y, de este modo, las principales ciudades latinoamericanas mantuvieron su liderazgo (Parnreiter, 2005). Las actividades relativas a los productores globales de servicios se ubicaron predominantemente en las últimas. Los estudios sobre las ciudades latinoamericanas y globalización se centran en las (i) ciudades mundiales (Kowarick y Campanario, 1986; Taschner y Bogus, 2001; Chion, 2002; Garza, 2005; Heron, 2005; Sánchez y Moura, 2005) o en las (ii) inversiones hechas en las regiones más desarrolladas de un país (Tolosa, 2003). La literatura carece de estudios sobre ciudades en proceso de mundialización. Tales ciudades se encuentran bajo el control de actores globales instalados en las ciudades mundiales. Éste es un nuevo parámetro que puede ser útil para las políticas en cuestión.

En el proceso de globalización contemporáneo, no siempre una ciudad que ejerce un importante

Programa de Engenharia de Produção, COPPE/ UFRJ, Rio de Janeiro, Brasil. E-mail: consoni@pep.ufrj.br.

Department of Geography, Loughborough University, Loughborough, UK. E-mail: P.J.Taylor@lboro.ac.uk.

** El Consejo Nacional Brasilero para el Desarrollo Científico y Tecnológico (CNPq) apoyó a Eliana Consoni Rossi, mientras que ESRC (Reino Unido) apoyó con beca la investigación de Peter Taylor que tuvo como resultado la metodología aplicada en este estudio. Enviado el 5 de septiembre de 2006, aprobado el 16 de febrero de 2007. papel en la red urbana nacional tiene el mismo desempeño en la red de ciudades mundiales. El ejemplo clásico es Washington. La inserción global de ciudades en esas condiciones es indirecta. En el caso de Washington, esto ocurre a través de Nueva York. Por otro lado, este mismo proceso indica que una ciudad de la red nacional, que también desempeña la función de ciudad mundial, puede ser interpretada como virtualmente expandida, tanto en el territorio nacional como lejos de él. Esa expansión corresponde a la hinterland y la outland de la referida ciudad, definidas, respectivamente, por el conjunto de ciudades domésticas y extranjeras, que reciben los representantes de los agentes urbanos globales.

Para contextualizar el proceso de globalización en las ciudades, en este estudio se recurre a dos temas ampliamente debatidos en la literatura. El primer tema, que se refiere a la definición de ciudad mundial; y el segundo, que se refiere a la estructura de la red de ciudades mundiales y a los estándares de red de los prestadores de servicio con alto valor agregado.

\section{Las ciudades en el proceso de globalización: estructura conceptual}

\section{La ciudad mundial}

La hipótesis de la ciudad mundial propuesta por Friedmann (1986) fue exhaustivamente trabajada por Sassen (1991/2001) en las ciudades de Londres, Nueva York y Tokio. De acuerdo con Sassen, la ciudad global es el centro de control del proceso contemporáneo de globalización, siendo la sede de empresas globales prestadoras de servicio con un alto valor agregado -servicios bancarios, legales, de contabilidad, propaganda, seguros, consultoría de gestión y otros-. Estas empresas operan como terceras, proveyendo servicios/informaciones especializadas y extremamente cualificadas. La meta de las empresas contratantes es minimizar costos y riesgos en sus estrategias de producción global. Estos agentes -empresas prestadoras de servicio con alto valor agregado- son estrictamente urbanos y aún se encuentran concentrados en pocas ciudades. De este modo, Sassen las denominó ciudades globales para diferenciarlas de las demás. Aunque en la literatura sobre la globalización contemporánea, 
el término mundial sea utilizado para designar esas ciudades (ver Friedmann, 1986), éste también fue usado para explicar otros procesos económicos mundiales, en diferentes períodos.

\section{La red de ciudades mundiales}

Según la hipótesis de Friedmann (1986), las ciudades mundiales operan en red. A partir de la estructura propuesta por Taylor $(2001 ; 2004)$ para la red de ciudades mundiales, los estudios basados en la red progresan. La estructura de Taylor se fundamenta en la organización de los agentes urbanos de la ciudad global de Sassen para atender a sus clientes. Según Taylor, los parámetros de la red de ciudades mundiales son tres: (i) los prestadores globales de servicio con alto valor agregado; (ii) las ciudades que componen sus redes; y (iii) el valor de cada ciudad de la red para cada uno de ellos. La superposición de estos valores configura la red de ciudades mundiales. Así, además de considerar la presencia del prestador de servicio en determinada ciudad, la metodología de Taylor estima el grado de autonomía de su principal representante local. Los parámetros son trabajados para obtener la conectividad de las ciudades mundiales en la red.

\section{Las redes de prestadores de servicio con alto valor agregado}

Antes de la globalización contemporánea, los prestadores de servicio, el bancario, por ejemplo, ya operaban en diversas ciudades del globo. Sin embargo, los servicios prestados eran limitados, siendo las actividades del sector manufacturero las que agregaban mayor valor a los productos. Luego, añadir valor a través de servicios es una de las características de la globalización actual. Las empresas prestadoras de servicios con alto valor agregado (APS) parecen haber implementado su estrategia de globalización en etapas, comprendiendo los nuevos mercados como extensas regiones y eligiendo la ciudad dominante de cada una de ellas. Son ejemplos, Hong Kong, en Asia, y San Pablo, en Latinoamérica. Gradualmente, los APS fueron ofreciendo servicios con alto valor agregado a otras ciudades. Por principio, los nódulos de la red de ciudades mundiales, coincidentes con las ciudades elegidas para localizar las sedes regionales, tuvieron el papel de articular las economías regionales/nacionales con la economía global (Friedmann, 1986; 1995).

Cada servicio con alto valor agregado tiene características diferenciadas de inserción global. Por ejemplo, los servicios bancarios son más fácilmente globalizados, ya que sus procesos son prácticamente iguales en todo el mundo. De esta manera, redes bancarias enteras son adquiridas de una sola vez. Considérese el caso del ingreso del HSBC y del ABN-AMRO, en Brasil, que adquirieron redes con más de 700 agencias. Por otro lado, los servicios legales son parcialmente globalizados, sea por las características intrínsecas, sea por las habilidades que se exigen de los profesionales abarcados, y debido a eso, estos servicios se encuentran en un reducido número de ciudades.

En el caso de los servicios legales, la velocidad de ampliación de su presencia global y de la penetración en nuevos territorios es menor que en los servicios bancarios. Cada servicio, en función del tipo de negocio, tiene más o menos opciones de tamaño de red. Por otra parte, en un nuevo territorio, los bancos pueden operar de tres formas: a través de una única agencia en una ciudad dominante, a través de una pequeña red en ciudades dominantes, o a través de una red nacional, que mezcla ciudades diversas. La última opción no es común en el caso de servicios legales.

\section{Gateway city}

El concepto de gateway city, discutido por primera vez por McKenzie (1933), auxilia al entendimiento del proceso de formación de la ciudad mundial que articula las economías global y regional/nacional. Ullman (1957) agregó al concepto la idea de que las gateway cities son la sede de empresas prestadoras de importantes servicios nacionales, en el caso, empresas de navegación y ferrovías. Burghardt (1971), al estudiar las ciudades fronterizas de los Estados Unidos, en el siglo XIX, las describió utilizando el término gateway city como un concepto de posición, caracterizándolas como un centro económico, que, además, funciona como entrada y salida para una extensa región, conectada a regiones distantes. Los estudios históricos (Drennan 1992; Burghardt 1971) colocan la gateway city como la antítesis de la ciudad tributaria en la teoría de los lugares centrales de 
Figura 1. Proceso de mundialización de ciudades.

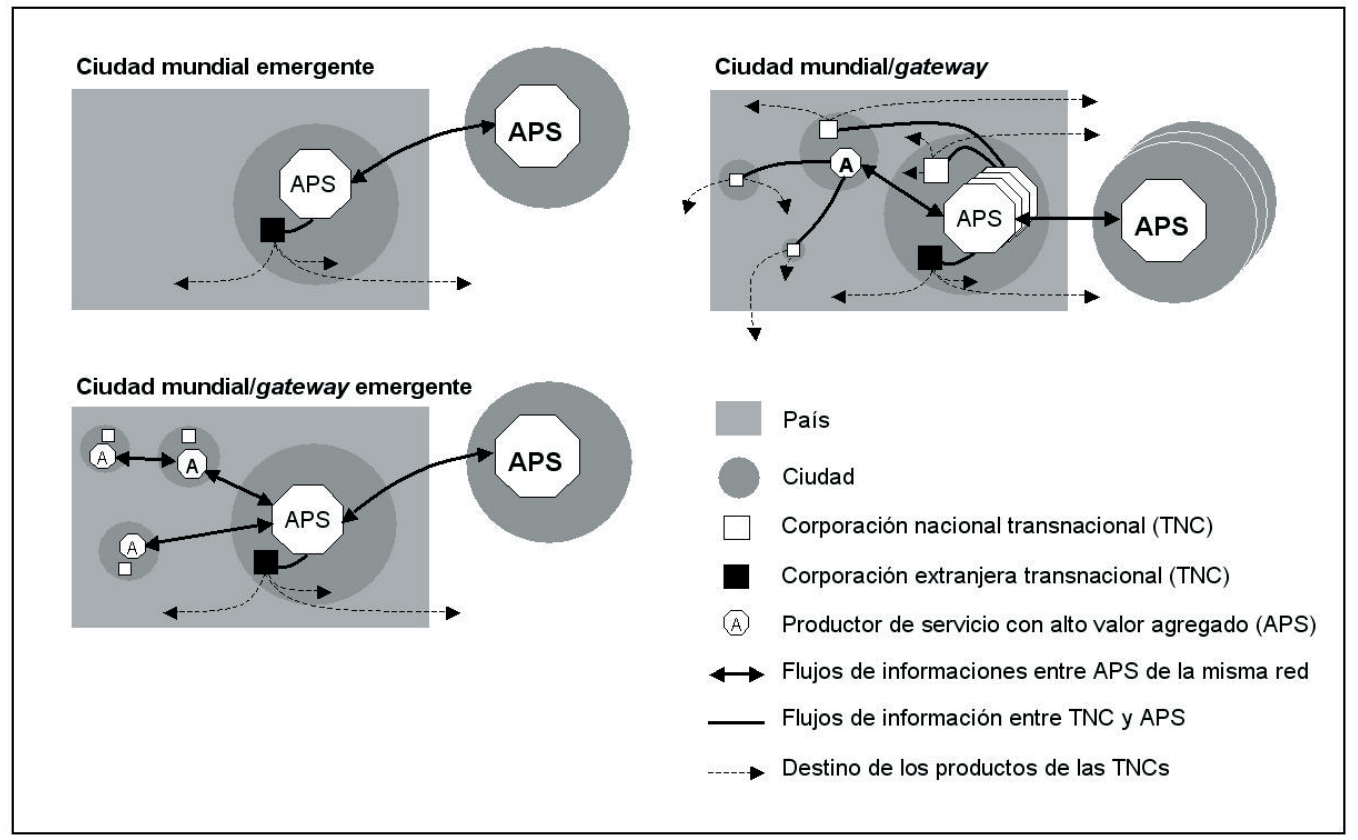

Fuente. Elaboración propia.

Christaller (1933). De acuerdo con Burghardt, las gateway cities son ciudades de contacto que actúan entre diferentes áreas homogéneas, como conexiones comerciales de larga distancia. Cuando funcionan como salida, esas urbes se extienden desde la hinterland que atienden hacia el exterior; por otro lado, la esencia de su función, la de entrada, es su lugar convergente en la malla de transportes. Las empresas americanas de aviación utilizan, hasta hoy, el término gateway city para referirse a la primera/última ciudad americana de un vuelo internacional. El autor de la idea que fue más allá de las definiciones asociadas a los transportes fue Pred (1977), que conceptualizó gateway city en función de los flujos de información más empleados entre ciudades que atienden sus hinterlands y mercados extranjeros distantes.

\section{Formación del territorio globalizado}

En este estudio, los flujos de informaciones, las bancarias, por ejemplo, más empleados en la gateway city, de Pred, son interpretados a través de esquemas. La Figura 1 muestra dos representaciones de estrategia de inserción de un determinado APS del sector bancario en un mercado todavía no atendido.
Ciudad mundial emergente. En el primer caso, el APS limita su estrategia de localización sólo a la ciudad dominante, que se convierte en una ciudad mundial emergente. Los flujos de información se restringen al representante localizado en la ciudad dominante. Ciertamente, la ciudad mundial emergente prestará servicio a otras ciudades. Sin embargo, tendrá una hinterland variable en el espacio y en el tiempo porque ella expresa el resultado de las necesidades de los contratantes de su servicio.

Ciudad mundial/gateway emergente. En el segundo caso, el APS tiene una estrategia de localización en red, incluyendo otras ciudades, además de la dominante, que se convierte, entonces, en una ciudad mundial/gateway emergente. Las demás ciudades de la red son emergentes en vía de tornarse mundiales. En el esquema de la derecha (Figura 1), tenemos una ciudad mundial que simultáneamente es una gateway city, pues, además de ser la sede de diversos APSs, por lo menos, uno de ellos optó por una estrategia de localización en red. En la gateway city, los flujos de informaciones se extienden por todas las ciudades de la red. La hinterland existe independientemente de las necesidades de las empresas contratantes. 
Cuadro 1. Ciudades mundiales de la muestra

\begin{tabular}{|l|l|l|l|}
\hline \multicolumn{1}{|c|}{ América del Norte } & \multicolumn{1}{|c|}{ Europa } & \multicolumn{1}{c|}{ Asia (Pacífico) } & \multicolumn{1}{c|}{ Otras regiones } \\
\hline Nueva York (2) & Londres (1) & Hong Kong (3) & Sydney (13) \\
\hline Chicago (7) & París (4) & Tokio (5) & San Pablo (16) \\
\hline Los Ángeles (9) & Milán (8) & Singapur (6) & México (18) \\
\hline Toronto (10) & Madrid (11) & Taipei (20) & Mumbai (21) \\
\hline San Francisco (17) & Ámsterdam (12) & Jacarta (22) & Buenos Aires (23) \\
\hline Miami (25) & Frankfurt (14) & & Melbourne (24) \\
\hline & Bruselas (15) & & \\
\hline & Zurich (19) & & \\
\hline
\end{tabular}

Nota: los números entre paréntesis corresponden a la clasificación del GaWC en 2000.

Fuente. Taylor (2004).

Es una hinterland permanente y explícita. En este caso, los flujos de informaciones bancarias más empleados pueden ser estudiados.

Este estudio complementa estudios precedentes (Rossi y Taylor, 2006; Rossi, Beaverstock y Taylor, 2007), y rescata el concepto de Pred para comprender la gateway city en el proceso de globalización contemporáneo. En el primer estudio, Rossi y Taylor se dedicaron al hueco dejado por investigadores que definieron gateway como una ciudad mundial con hinterland ignorada (Grant, 2001; Grant y Nijman 2002). En el segundo estudio, fueron investigadas las relaciones intra e interurbanas creadas por las necesidades de los contratantes de servicios con alto valor agregado en Brasil, en el período de recolección de los datos. El énfasis estuvo en la hinterland variable, apuntada en el parágrafo anterior. El aporte del presente estudio está en la focalización de las ciudades más importantes de la red nacional y sus participaciones en las redes de gateway city brasileñas. Los flujos de los servicios bancarios es la base de este análisis, por las razones ya expuestas.

En resumen, primero se presentan las hipótesis del presente estudio. Después se describen las metodologías de recolección y análisis de los datos, que se basan respectivamente en la metodología de "Globalization and World City study group and network" (GaWC) y en el análisis de componentes principales, un recurso de análisis estadístico multivariado. En seguida, se presentan el contenido y los resultados de la investigación y, finalmente, la conclusión.

\section{Hipótesis principales}

La primera hipótesis se relaciona a una de las funciones señaladas por Friedmann $(1986,1995)$ para la ciudad mundial: articular las economías nacional y global. Luego, ¿la ciudad de San Pablo estaría ejerciendo esta función? La segunda hipótesis se halla volcada hacia el patrón de las ciudades involucradas en el proceso: ‘el hecho de que la ciudad sea la capital administrativa del país (Brasilia) o un importante polo industrial (Manaos) predispone favorablemente su inserción en la red regional de ciudades articulada por la metrópoli mundial (San Pablo)? Los resultados de la segunda hipótesis pueden auxiliar a quienes toman las decisiones en la formulación de políticas.

\section{Metodología y datos utilizados en la investigación}

El presente estudio se origina de una investigación sobre la inserción de ciudades brasileñas en la red de ciudades mundiales. Para su realización, fue empleado el método cuantitativo de recolección y análisis, sin embargo, parte de los datos se han conseguido a través de un cuestionario semiestructurado. Los datos fueron trabajados para obtener las siguientes informaciones: (i) las ciudades brasileñas articuladoras de las economías regional/nacional y global; y (ii) las hinterlands de estas ciudades. Para este fin, utilizamos el método estadístico multivariado de análisis de componentes principales en una matriz de ciudades versus prestadores de servicios bancarios, organizada según la metodología del GaWC. 


\section{Muestras de ciudades y de bancos}

Cuadro 2. Ciudades brasileñas de la muestra

\begin{tabular}{|l|l|}
\hline \multicolumn{1}{|c|}{ Ciudad-región } & \multicolumn{1}{c|}{ Ciudad aislada } \\
\hline Belo Horizonte (3) & Campo Mourao (25) \\
\hline Belén (20) & Chapecó (24) \\
\hline Blumenau (13) & Concordia (18) \\
\hline Brasilia (5) & Manaos (10) \\
\hline Campinas (8) & Montes Claros (29) \\
\hline Caxias do Sul (21) & Piracicaba (27) \\
\hline Curitiba (7) & Poços de Caldas (22) \\
\hline Florianópolis (14) & Uberlandia (15) \\
\hline Fortaleza (16) & \\
\hline Goiania (17) & \\
\hline Joinville (19) & \\
\hline Mogi Guaçu (28) & \\
\hline Porto Alegre (4) & \\
\hline Recife (11) & \\
\hline Ribeirao Preto (26) & \\
\hline Río de Janeiro (2) & \\
\hline Salvador (6) & \\
\hline Santos (30) & \\
\hline San José dos Campos (12) & \\
\hline San Pablo (1) & \\
\hline Sorocaba (23) & \\
\hline Victoria (9) & \\
\hline
\end{tabular}

Nota: los números entre paréntesis corresponden a la clasificación por ventas acumuladas en 1996-2002.

Fuente. Rossi y Taylor (2005)

La muestra de ciudades se compone de las 25 principales ciudades mundiales (Cuadro $1 \mathrm{y}$ Figura 2) identificadas por Taylor (2004), y las primeras 30 ciudades brasileñas (ciudad aislada y ciudad-región), sedes de las 500 mayores empresas existentes en Brasil, según la revista brasileña de negocios Exame Melhores e Maiores (Abril, 1996-2003; 2003b), en el período de 1996 a 2002 (Cuadro 2 y Figura 3). El ciclo fue elegido porque comienza cuando los bancos extranjeros se instalaron en Brasil, operando conforme el proceso de globalización (Carvalho, 2000). En esos siete años, fueron relacionadas 868 empresas y éstas se ubicaron en 167 ciudades. Los criterios para agrupar ciudades aisladas en ciudad-región y para clasificar las ciudades fueron, respectivamente, los agrupamientos dados por el estudio de IPEA,
IBGE y UNICAMP (2001) sobre la red urbana brasileña y las ventas acumuladas de las empresas de cada ciudad en el período, utilizando como fuente la revista Exame Melhores e Maiores (Abril, 1996-2003; 2003b). La muestra final tiene 54 ciudades porque San Pablo es una de las 25 ciudades mundiales seleccionadas.

La muestra de bancos se basó en tres fuentes: los 50 mayores bancos en operación en Brasil, durante el 2002, señalados por la revista Exame Melhores e Maiores (Abril, 2003a; 2003b) y clasificados por su patrimonio líquido ajustado; la página del Banco Central de Brasil en Internet, cuyo banco de datos informó (i) la clasificación de los bancos en el Sistema Financiero Brasileño, en diciembre de 2003, (ii) el grupo al cual cada banco pertenece, y (iii) para cada banco, las ciudades de su red de agencias. La tercera fuente de datos fue la página de los bancos en Internet, donde (i) fueron confirmadas las ciudades de la red de cada banco obtenida en el Banco Central de Brasil; (ii) fue identificada la extensión internacional de cada banco, es decir, las ciudades donde operan fuera de Brasil, y (iii) para cada red bancaria, el nivel de autonomía del principal agente de cada ciudad de la muestra -sede mundial, sede nacional, agencia o autoservicio-. La selección inicial comprende los 50 mayores bancos. Se agruparon los datos de los bancos pertenecientes a la misma corporación y fueron eliminados aquellos que no estuvieron presentes en por lo menos 4 de las 54 ciudades de la muestra. 19 bancos fueron absorbidos por el agrupamiento, o no se calificaron. La selección final tuvo 31 bancos.

\section{Metodología}

La matriz de ciudades versus bancos se estructuró de acuerdo con la metodología desarrollada por Taylor (2004) y de acuerdo con los estudios del GaWC. Fue aplicada una codificación que señala la relevancia de la agencia principal de cada banco en la ciudad, de la siguiente forma: 4 para la ciudad que es sede mundial, 3 para cada ciudad sede en Brasil, 2 para la ciudad que tenga por lo menos una agencia, 1 para la ciudad que, por lo menos, cuenta con autoservicio. Las ciudades que no presentaron ninguna de las condiciones anteriores, se codificaron como 0 . De esta forma, 1674 valores fueron creados para producir la matriz final de la 
Figura 2. Posición relativa de las ciudades mundiales de la muestra.

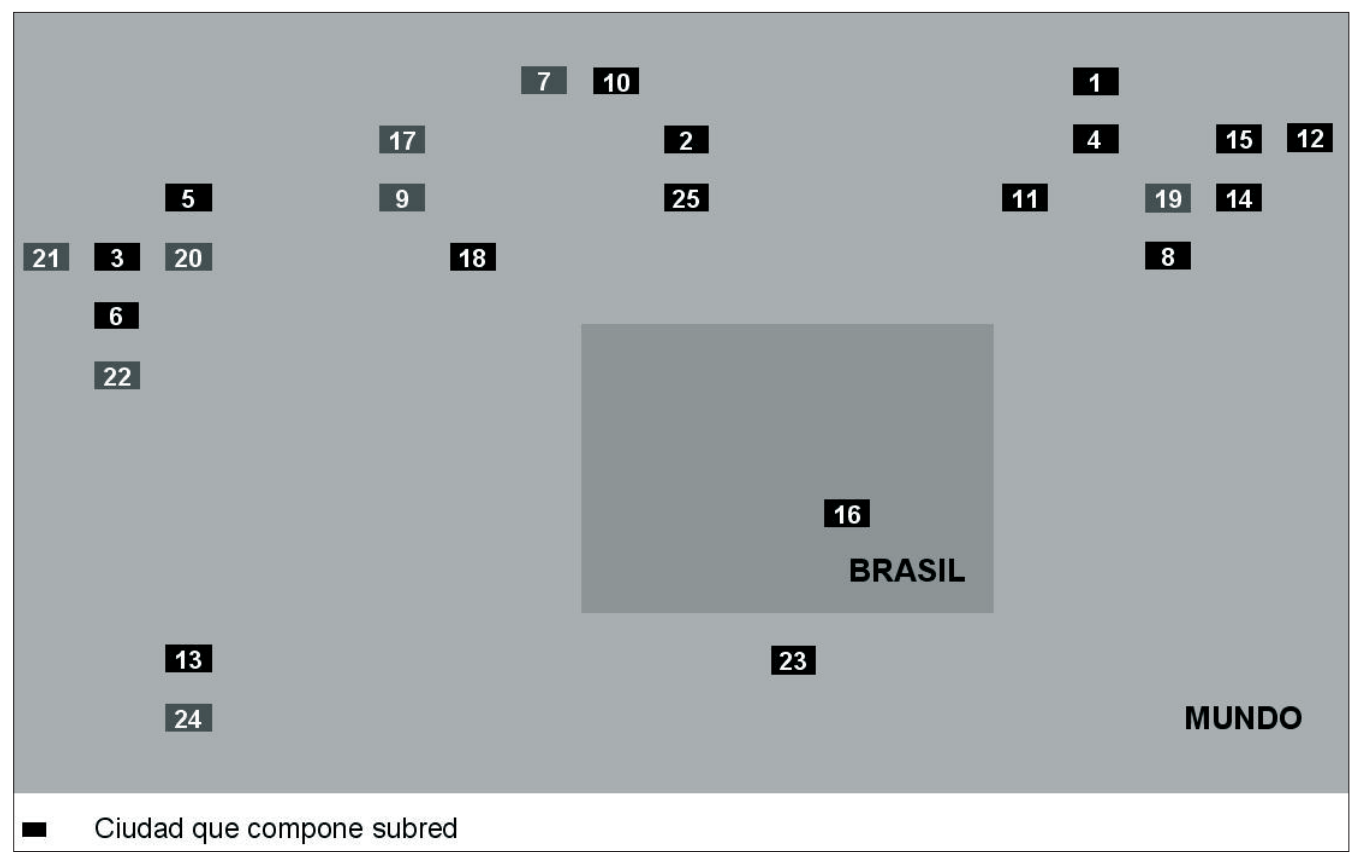

1 Londres, 2 Nueva York, 3 Hong Kong, 4 París, 5 Tokio, 6 Singapur, 7 Chicago, 8 Milán, 9 Los Ángeles, 10 Toronto, 11 Madrid, 12 Ámsterdam, 13 Sydney, 14 Frankfurt, 15 Bruselas, 16 San Pablo, 17 San Francisco, 18 México, 19 Zurich, 20 Taipei, 21 Mumbai, 22 Jacarta, 23 Buenos Aires, 24 Melbourne, 25 Miami.

Nota: los números corresponden a la clasificación del GaWC en 2000.

Fuente. Elaboración propia, con base en Taylor (2004).

actividad bancaria, 54 ciudades por 31 bancos. Las columnas de la matriz de datos representan la estrategia de localización de cada banco, o sea, las ciudades en que operan y el nivel de operación. Cada línea representa el conjunto bancario del que cada ciudad dispone, tanto en términos de los bancos que en ella operan, como el nivel de las actividades para atender a sus clientes.

\section{Análisis de componentes principales}

El análisis de componentes principales (PCA) es una de las formas de hacer análisis factorial, uno de los métodos de la estadística multivariada. Esa técnica es empleada para reducir extensas matrices de datos a sus factores básicos, en este caso, componentes básicos. El modelo de la PCA es el más simple y con el menor bagaje teórico (Rummel, 1970), empleado con éxito en anteriores análisis de este tipo (Taylor y Walker, 2001; Taylor, Catalano y Walker, 2002 y 2004; Taylor, 2004). El modelo utilizado, en ese estudio, es el PCA con rotación varimax, ideal para garantizar resultados claros e interpretables.
El PCA consiste en representar las coordenadas de un conjunto de datos en un otro sistema de ejes más conveniente para el análisis. Esas nuevas coordenadas son el resultado de la combinación lineal de las variables originales, y son representadas sobre ejes ortogonales, obtenidos en orden decreciente de varianza. Así, el primer componente retiene más informaciones que el segundo, que no retiene las informaciones contabilizadas anteriormente. Los nuevos ejes se mantienen ortogonales, garantizando que los componentes no se correlacionan. Cada nuevo componente es una variable compuesta, que agrupa variables originales con base en sus interrelaciones. Entonces, los componentes son grupos de variables semejantes. En este caso, existen 31 variables: las 31 estrategias de localización de los bancos líderes, que fueron reducidas a seis componentes. Cada uno de los componentes corresponde a un grupo de bancos con estrategia de localización y nivel de atendimiento semejantes. Cada componente fue interpretado como una estrategia de localización compuesta o común, para un dado grupo de bancos. 
Figura 3. Posición relativa de las ciudades brasileñas de la muestra.

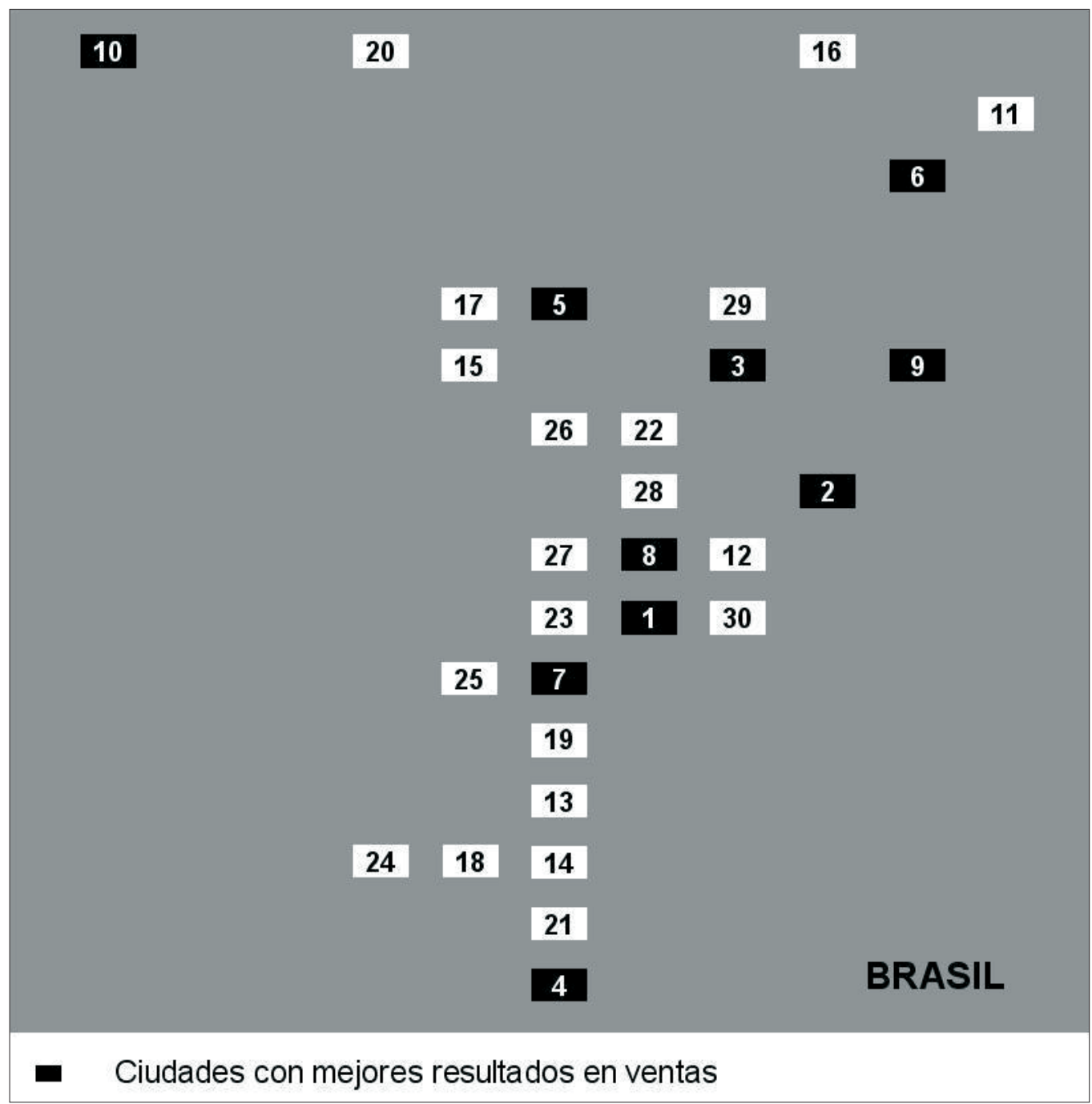

1 San Pablo, 2 Río de Janeiro, 3 Belo Horizonte, 4 Porto Alegre, 5 Brasilia, 6 Salvador, 7 Curitiba, 8 Campinas, 9 Victoria, 10 Manaos, 11 Recife, 12 San José dos Campos, 13 Blumenau, 14 Florianópolis, 15 Uberlandia, 16 Fortaleza, 17 Goiania, 18 Concordia, 19 Joinville, 20 Belén, 21 Caxias do Sul, 22 Poços de Caldas, 23 Sorocaba, 24 Chapecó, 25 Campo Mourao, 26 Ribeirao Preto, 27 Piracicaba, 28 Mogi Guaçu, 29 Montes Claros, 30 Santos.

Nota: los números corresponden a la clasificación por ventas acumuladas entre 1996 y 2002.

Fuente. Elaboración propia, con base en Rossi y Taylor (2005).

Tres informaciones deben ser observadas en el PCA, pues ellas ayudan a interpretar cada componente: (i) Las variables agrupadas (bancos), (ii) la puntuación de los objetos presentes (ciudades), y (iii) la medida de importancia de cada componente (varianza). Las variables con mayor peso en el agrupamiento (bancos) definen el grupo de variables que forman un componente, haciendo explícita la estrategia de localización. La puntuación muestra cómo el componente está distribuido a través de los objetos (ciudades); los valores de la puntuación son estandarizados, o sea tienen media cero, y la mayor parte está entre más o menos dos. Las ciudades con mayor puntuación en un componente describen la estrategia de localización. En este estudio, el conjunto de las ciudades destacadas positivamente en cada componente fue identificado como una subred en la red general de ciudades, 
correspondiendo a la hinterland y a la outland de la urbe que lidera el componente. La varianza de los datos de los bancos agrupados por la variación del conjunto de datos determina la importancia del componente. La suma de esas proporciones es la varianza mantenida al hacerse la reducción para componentes. Usualmente, la reducción mantiene el $50 \%$ de la varianza original.

Determinar el número de componentes es un procedimiento clave del PCA. No hay un recurso de aceptación general que ampare esta elección. Fue empleado el método experimental para encontrar las variables particularmente estables, aquéllas que siempre aparecen en varias opciones de número de componentes (Taylor et al., 2002). El segundo criterio empleado limitó el número de las mismas al mínimo, siempre y cuando las primeras diez ciudades brasileñas de la muestra (Figura 3) se encuentren destacadas positivamente (ver Cuadro 2). Los dos criterios resultarán en seis componentes: seis estrategias de localización.

\section{Descripción del contenido y resultados de la investigación}

Las seis estrategias de localización de los bancos líderes en operación en Brasil fueron resumidas en los Cuadros 3a, 3b, 4a y 4b. Los resultados de esos cuadros se presentan agrupados en cuatro temas, a seguir. El primero identifica las estrategias a través de la distribución geográfica de las ciudades destacadas positivamente -las subredes-. El segundo trata del significado de las agrupaciones y las puntuaciones negativas. El tercero destaca las gateway cities brasileñas. El cuarto trata del desempeño de las diez principales ciudades brasileñas, clasificadas de acuerdo a su producción en ventas en las estrategias de localización de los bancos.

\section{Las subredes}

Las subredes relacionadas en los Cuadros $3 \mathrm{a}$ y $3 \mathrm{~b}$ configuran flujos independientes de información bancaria. Primero, se interpretan las estrategias de I a IV, que presentan una varianza más expresiva (Figura 4).

Aunque se habían agrupado bancos brasileños y extranjeros, las estrategias I y IV sólo destacaron ciudades brasileñas. La primera se concentró en las ciudades brasileñas, sedes nacionales de los bancos seleccionados. ${ }^{1}$ La segunda, convergió para las ciudades del núcleo económico brasileño. Ambas son lideradas por San Pablo.

Agrupando apenas bancos extranjeros, la estrategia II se concentró en las dos más importantes ciudades brasileñas, formando una subred de éstas con 14 ciudades mundiales, además de San Pablo, que, a la vez, lidera la subred.

La estrategia III es la única en la cual una ciudad no brasileña recibe la puntuación máxima. Este resultado, sin duda, refleja la importancia de Nueva York para los bancos en general, sobre todo para los brasileños, una vez que siete de los diez bancos agrupados son de Brasil. Entonces, la estrategia III no está explícitamente liderada por Nueva York, sino enfocada en Nueva York. Esta subred destaca las ciudades mundiales de la muestra que están conectadas a las ciudades del Brasil meridional, incluyendo Buenos Aires, la ciudad mundial más cercana. Aunque no lidere la subred, la ciudad brasileña con mayor puntuación fue San Pablo.

Las dos últimas estrategias son menores, consideradas sus varianzas (Cuadro 3b). Fueron representadas en la Figura 5. En la estrategia V, sólo dos bancos extranjeros se agruparon, creando una subred liderada por Curitiba, cuya elevada puntuación positiva se diferenció de todas las otras puntuaciones de ciudades líderes del conjunto de subredes (Cuadros 3a y 3b). Esta estrategia tiene otra característica notable: excluye a San Pablo (Cuadro 4b). Además de eso, es una estrategia global que incluye la más importante ciudad mundial de la muestra, Londres, sede mundial de uno de los bancos agrupados -HSBC-. Aunque la red de ciudades de este banco sea la mayor, abarcando 52 ciudades de la muestra, en esa estrategia apenas se destacan las ciudades comunes a las redes de los dos bancos agrupados. Son importantes ciudades brasileñas del sector agrícola, núcleo del negocio del otro banco.

\footnotetext{
Salvador, en 2002, fue sede nacional del BANEB, banco del grupo Bradesco, y, por eso, en la matriz final, tuvo sus datos absorbidos en la columna correspondiente al principal banco del grupo, Bradesco, manteniendo la puntuación de Salvador como sede nacional del BANEB.
} 
Cuadro 3a. Estrategias de localización de los bancos. Estrategias I y II.

\begin{tabular}{|c|c|c|c|c|c|}
\hline \multirow[t]{2}{*}{ Estrategia } & \multirow{2}{*}{$\begin{array}{c}\text { Varianza } \\
\%\end{array}$} & \multicolumn{2}{|c|}{ Principales agrupamientos de bancos } & \multicolumn{2}{|c|}{ Principales puntuaciones de ciudades } \\
\hline & & Agrupamientos & Agentes & Puntuación & Subred \\
\hline $\mathrm{I}$ & 23 & 0,84 & BMG & 2,82 & San Pablo (1) \\
\hline & & 0,82 & Santos & 2,79 & Río de Janeiro (2) \\
\hline & & 0,76 & BIC & 2,33 & Belo Horizonte (3) \\
\hline & & 0,74 & Nordeste & 2,04 & Salvador $(6)$ \\
\hline & & 0,73 & BBA & 2,03 & Porto Alegre (4) \\
\hline & & 0,72 & IBI & 1,96 & Brasilia (5) \\
\hline & & 0,7 & Banrisul & 1,43 & Recife (11) \\
\hline & & 0,67 & Pactual & 1,32 & Fortaleza (16) \\
\hline & & 0,6 & BASA & 1,25 & Curitiba (7) \\
\hline & & 0,59 & Rural & 0,75 & Belén (20) \\
\hline & & 0,55 & Safra & & \\
\hline & & 0,55 & BankBoston & & \\
\hline & & 0,49 & GM & & \\
\hline & & 0,43 & Mercantil do Brasil & & \\
\hline & & 0,42 & Bradesco & & \\
\hline & & 0,42 & Unibanco & & \\
\hline & & 0,4 & Itaú & & \\
\hline & & 0,4 & CEF & & \\
\hline & & 0,37 & Citibank & & \\
\hline II & 23 & 0,88 & Tokio Mitsubishi & 2,38 & San Pablo (1) \\
\hline & & 0,86 & WestLB Brasil & 1,76 & Londres (1) \\
\hline & & 0,82 & Deutsche Bank & 1,76 & Nueva York (2) \\
\hline & & 0,73 & BNP Paribas & 1,73 & Tokio (5) \\
\hline & & 0,72 & Citibank & 1,72 & París (4) \\
\hline & & 0,71 & Sumitomo & 1,55 & Frankfurt (14) \\
\hline & & 0,64 & ABC Brasil & 1,41 & Singapur (6) \\
\hline & & & & 1,3 & Madrid (11) \\
\hline & & & & 1,15 & Milán (8) \\
\hline & & & & 1,07 & Buenos Aires (23) \\
\hline & & & & 1,02 & Bruselas (15) \\
\hline & & & & 0,94 & Hong Kong (3) \\
\hline & & & & 0,82 & México (18) \\
\hline & & & & 0,81 & Río de Janeiro (2) \\
\hline & & & & 0,78 & Toronto (10) \\
\hline & & & & 0,51 & Sydney (13) \\
\hline
\end{tabular}

Nota: los números entre paréntesis corresponden a la posición de la ciudad en las clasificaciones de las ciudades brasileñas de la muestra y de las ciudades mundiales.

Fuente. Elaboración propia.

La última estrategia también agrupa pocos bancos. Uno brasileño y dos extranjeros. Fue el banco brasileño, BASA, el que puso en evidencia las dos principales ciudades del norte de Brasil. La subred es liderada por Belén, que es la sede del banco brasileño. Las puntuaciones configuran una subred de ciudades del Brasil septentrional, polarizadas por el norte y por el sudeste y mezcladas con ciudades mundiales europeas y una canadiense.

\section{Significado de los resultados negativos}

Podemos visualizar un componente como dos conjuntos de bancos y ciudades. En el conjunto positivo (Cuadros $3 \mathrm{a}$ y $3 \mathrm{~b}$ ) se encuentran los elementos que efectivamente constituyen el componente. En el conjunto negativo (Cuadros $4 \mathrm{a}$ y $4 \mathrm{~b}$ ) se encuentran los elementos que se contraponen a ellos. Así, los agrupamientos y puntuaciones 
Cuadro 3b. Estrategias de localización de los bancos. Estrategias III, IV, V y VI.

\begin{tabular}{|c|c|c|c|c|c|}
\hline \multirow[t]{2}{*}{ Estrategia } & \multirow{2}{*}{$\begin{array}{l}\text { Varianza } \\
\%\end{array}$} & \multicolumn{2}{|c|}{ Principales agrupamientos de bancos } & \multicolumn{2}{|c|}{ Principales puntuaciones de ciudades } \\
\hline & & Agrupamientos & Agentes & Puntuación & Subred \\
\hline \multirow[t]{15}{*}{ III } & 12 & 0,83 & Santander & 1,88 & Nueva York (2) \\
\hline & & 0,57 & Itaú & 1,2 & Londres (1) \\
\hline & & 0,55 & Bradesco & 1,16 & San Pablo (1) \\
\hline & & 0,41 & BankBoston & 1,09 & Madrid (11) \\
\hline & & 0,41 & GM & 1 & Chapecó (24) \\
\hline & & 0,4 & $\mathrm{CEF}$ & 0,83 & Porto Alegre (4) \\
\hline & & 0,37 & Safra & 0,82 & Joinville (19) \\
\hline & & & & 0,67 & Poços de Caldas (22) \\
\hline & & & & 0,66 & Caxias do Sul (21) \\
\hline & & & & 0,66 & Campo Mourao (25) \\
\hline & & & & 0,66 & Florianópolis (14) \\
\hline & & & & 0,64 & Tokio (5) \\
\hline & & & & 0,6 & Brasilia (5) \\
\hline & & & & 0,59 & Milán (8) \\
\hline & & & & 0,53 & Miami (25) \\
\hline \multirow[t]{6}{*}{ IV } & 8 & 0,86 & Nossa Caixa & 2,83 & San Pablo (1) \\
\hline & & 0,51 & IBI & 2,68 & Campinas (8) \\
\hline & & & & 1,28 & Mogi Guaçu (28) \\
\hline & & & & 1,15 & Santos (30) \\
\hline & & & & 1,03 & Belo Horizonte (3) \\
\hline & & & & 0,73 & Curitiba (7) \\
\hline \multirow[t]{5}{*}{$\mathrm{V}$} & 5 & 0,84 & CNH Capital & 5,53 & Curitiba (7) \\
\hline & & 0,6 & HSBC & 1,63 & Londres (1) \\
\hline & & & & 1,5 & Goiania (17) \\
\hline & & & & 1,37 & Ribeirao Preto (26) \\
\hline & & & & 1,09 & Uberlandia (15) \\
\hline \multirow[t]{9}{*}{ VI } & 5 & 0,77 & ABN-AMRO (Real) & 2,43 & Belén (20) \\
\hline & & 0,44 & BASA & 2,42 & Ámsterdam (12) \\
\hline & & 0,34 & HSBC & 1,84 & Manaos (10) \\
\hline & & & & 1,57 & San Pablo (1) \\
\hline & & & & 1,08 & Londres (1) \\
\hline & & & & 1,01 & París (4) \\
\hline & & & & 0,71 & Milán (8) \\
\hline & & & & 0,66 & Victoria (9) \\
\hline & & & & 0,64 & Santos (30) \\
\hline
\end{tabular}

Nota: los números entre paréntesis corresponden a la posición de la ciudad en las clasificaciones de las ciudades brasileñas de la muestra y de las ciudades mundiales.

Fuente. Elaboración propia. 
Eliana Consoni Rossi y Peter J. Taylor

Cuadro 4a. Bancos y ciudades discriminados en cada estrategia. Estrategias I y II.

\begin{tabular}{|c|c|c|c|c|c|}
\hline \multirow{2}{*}{ Estrategia } & \multirow{2}{*}{$\begin{array}{c}\text { Varianza } \\
\%\end{array}$} & \multicolumn{2}{|c|}{ Principales agrupamientos de bancos } & \multicolumn{2}{|c|}{ Principales puntuaciones de ciudades } \\
\hline & & Agrupamientos & Agentes & Puntuación & Subred \\
\hline \multirow[t]{17}{*}{ I } & 23 & & & $-0,5$ & Hong Kong \\
\hline & & & & $-0,52$ & Sydney \\
\hline & & & & $-0,54$ & Toronto \\
\hline & & & & $-0,55$ & Chapecó \\
\hline & & & & $-0,58$ & Ámsterdam \\
\hline & & & & $-0,67$ & Frankfurt \\
\hline & & & & $-0,75$ & Londres \\
\hline & & & & $-0,79$ & Madrid \\
\hline & & & & $-0,81$ & París \\
\hline & & & & $-0,88$ & Milán \\
\hline & & & & $-0,88$ & Campo Mourao \\
\hline & & & & $-0,89$ & Uberlandia \\
\hline & & & & $-0,9$ & Poços de Caldas \\
\hline & & & & $-0,98$ & Sorocaba \\
\hline & & & & $-0,98$ & Piracicaba \\
\hline & & & & $-1,02$ & San José dos Campos \\
\hline & & & & $-1,18$ & Mogi Guaçu \\
\hline \multirow[t]{23}{*}{ II } & 23 & $-0,4$ & Safra & $-0,53$ & Salvador \\
\hline & & $-0,47$ & Rural & $-0,75$ & Concordia \\
\hline & & $-0,48$ & GM & $-0,78$ & Chapecó \\
\hline & & $-0,53$ & Bradesco & $-0,79$ & Campo Mourao \\
\hline & & $-0,56$ & Mercantil do Brasil & $-0,8$ & San José dos Campos \\
\hline & & $-0,58$ & Itaú & $-0,8$ & Ribeirao Preto \\
\hline & & $-0,61$ & Unibanco & $-0,86$ & Blumenau \\
\hline & & $-0,75$ & $\mathrm{CEF}$ & $-0,86$ & Brasilia \\
\hline & & & & $-0,86$ & Mogi Guaçu \\
\hline & & & & $-0,9$ & Poços de Caldas \\
\hline & & & & $-0,97$ & Fortaleza \\
\hline & & & & $-0,98$ & Florianópolis \\
\hline & & & & $-0,99$ & Uberlandia \\
\hline & & & & $-1,02$ & Manaos \\
\hline & & & & $-1,03$ & Sorocaba \\
\hline & & & & $-1,03$ & Piracicaba \\
\hline & & & & $-1,05$ & Santos \\
\hline & & & & $-1,06$ & Joinville \\
\hline & & & & $-1,09$ & Caxias do Sul \\
\hline & & & & $-1,1$ & Victoria \\
\hline & & & & $-1,12$ & Goiania \\
\hline & & & & $-1,19$ & Montes Claros \\
\hline & & & & $-1,21$ & Belén \\
\hline
\end{tabular}

Fuente. Elaboración propia. 
Cuadro 4b. Bancos y ciudades discriminados en cada estrategia. Estrategias III, IV, V y VI.

\begin{tabular}{|c|c|c|c|c|c|}
\hline \multirow[t]{2}{*}{ Estrategia } & \multirow{2}{*}{$\begin{array}{c}\text { Varianza } \\
\%\end{array}$} & \multicolumn{2}{|c|}{ Principales agrupamientos de bancos } & \multicolumn{2}{|c|}{ Principales puntuaciones de ciudades } \\
\hline & & Agrupamientos & Agentes & Puntuación & Subred \\
\hline \multirow[t]{14}{*}{ III } & 12 & & & $-0,57$ & Montes Claros \\
\hline & & & & $-0,75$ & Brúcelas \\
\hline & & & & $-1,01$ & Toronto \\
\hline & & & & $-1,09$ & Belén \\
\hline & & & & $-1,22$ & Sydney \\
\hline & & & & $-1,28$ & Zurich \\
\hline & & & & $-1,35$ & Ámsterdam \\
\hline & & & & $-1,48$ & Los Ángeles \\
\hline & & & & $-1,73$ & Chicago \\
\hline & & & & $-1,74$ & San Francisco \\
\hline & & & & $-1,86$ & Melbourne \\
\hline & & & & $-2,01$ & Jacarta \\
\hline & & & & $-2,02$ & Taipei \\
\hline & & & & $-2,02$ & Mumbai \\
\hline \multirow[t]{16}{*}{ IV } & 8 & & & $-0,5$ & Salvador \\
\hline & & & & $-0,52$ & Ámsterdam \\
\hline & & & & $-0,54$ & Zurich \\
\hline & & & & $-0,6$ & México \\
\hline & & & & $-0,67$ & Miami \\
\hline & & & & $-0,72$ & Buenos Aires \\
\hline & & & & $-0,79$ & Londres \\
\hline & & & & $-0,8$ & Madrid \\
\hline & & & & $-0,8$ & Chapecó \\
\hline & & & & $-0,97$ & Concordia \\
\hline & & & & $-0,99$ & Belén \\
\hline & & & & $-1,09$ & Manaos \\
\hline & & & & $-1,15$ & Fortaleza \\
\hline & & & & $-1,25$ & Florianópolis \\
\hline & & & & $-1,94$ & Porto Alegre \\
\hline & & & & $-1,99$ & Brasilia \\
\hline \multirow[t]{12}{*}{$\mathrm{V}$} & 5 & & & $-0,5$ & Poços de Caldas \\
\hline & & & & $-0,51$ & Chapecó \\
\hline & & & & $-0,53$ & Campo Mourao \\
\hline & & & & $-0,54$ & Manaos \\
\hline & & & & $-0,67$ & Concordia \\
\hline & & & & $-0,67$ & Recife \\
\hline & & & & $-0,77$ & Belén \\
\hline & & & & $-0,92$ & Montes Claros \\
\hline & & & & $-1,15$ & Río de Janeiro \\
\hline & & & & $-1,25$ & San Pablo \\
\hline & & & & $-1,58$ & Chicago \\
\hline & & & & $-1,86$ & Los Ángeles \\
\hline \multirow[t]{13}{*}{ VI } & 5 & $-0,34$ & Citibank & $-0,53$ & Blumenau \\
\hline & & & & $-0,65$ & Campinas \\
\hline & & & & $-0,7$ & Melbourne \\
\hline & & & & $-0,71$ & Belo Horizonte \\
\hline & & & & $-0,74$ & Buenos Aires \\
\hline & & & & $-0,91$ & Chicago \\
\hline & & & & $-0,97$ & Recife \\
\hline & & & & $-1,03$ & New York \\
\hline & & & & $-1,04$ & Salvador \\
\hline & & & & $-1,86$ & San Francisco \\
\hline & & & & $-2,11$ & Concordia \\
\hline & & & & $-2,65$ & Miami \\
\hline & & & & $-2,73$ & Los Ángeles \\
\hline
\end{tabular}

Fuente. Elaboración propia. 
Figura 4. Principales estrategias de localización de los bancos de la muestra.

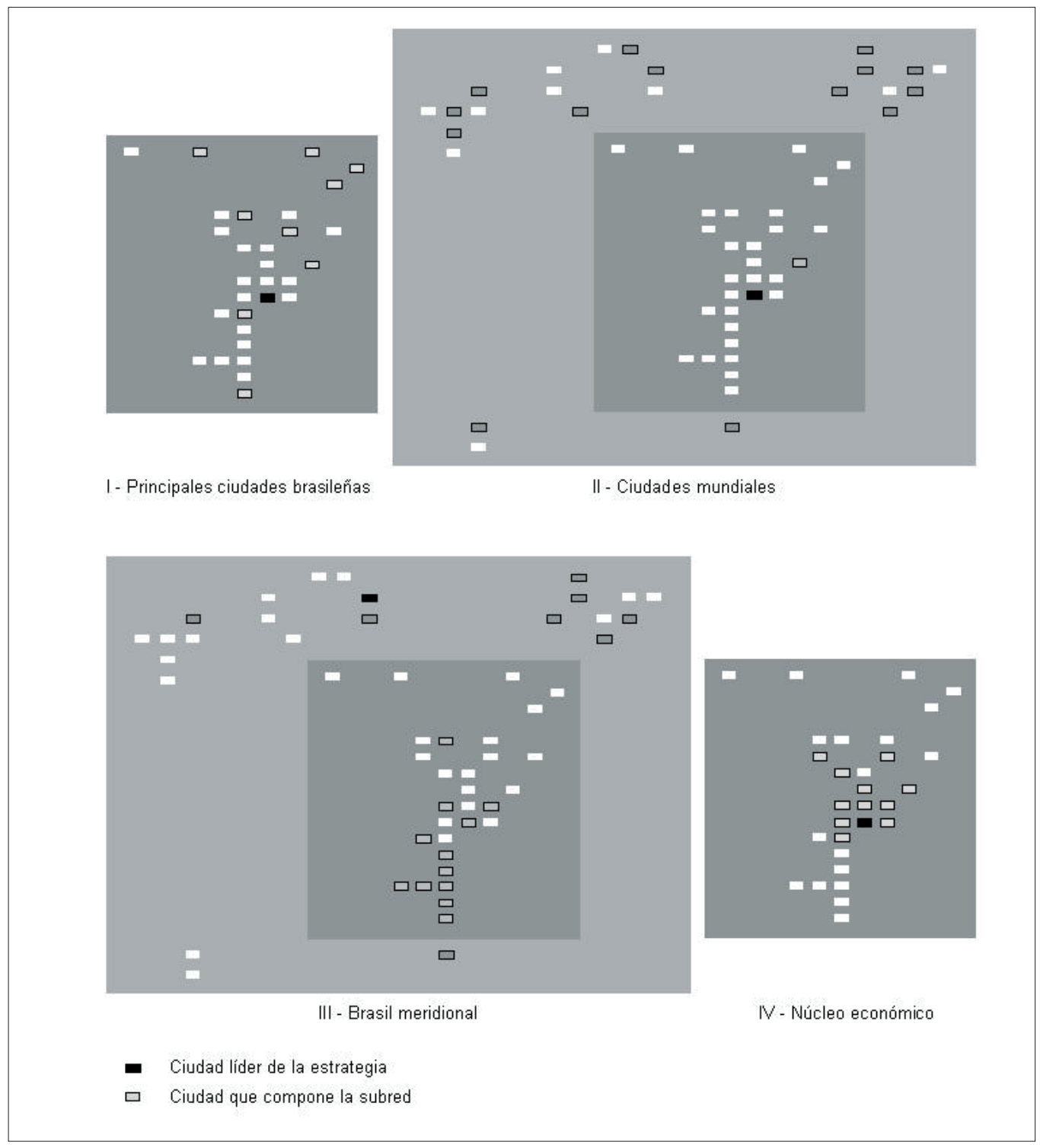

Nota: ver identificación de las ciudades en las Figuras 2 y 3 .

Fuente. Elaboración propia.

negativas relacionan los bancos y las ciudades declaradamente excluidos del componente. Surge de ahí la importancia de analizar este conjunto.

Los Cuadros 4a y 4b muestran que sólo dos componentes tuvieron agrupamiento negativo de los bancos. El primero de ellos corresponde a la estrategia de localización de los bancos globales -la estrategia II- en la cual los bancos excluidos son brasileños, sin agencias en las ciudades mundiales de la muestra. El segundo es la estrategia de localización polarizada en las regiones norte y sudeste -la estrategia VI-, enfocada en el Brasil septentrional. El mayor banco americano -Citibank- desprecia, de forma notoria, esta estrategia. Este hecho se refuerza por la puntuación muy negativa de las ciudades americanas en la estra- 
Figura 5. Estrategias menores de localización de los bancos de la muestra.

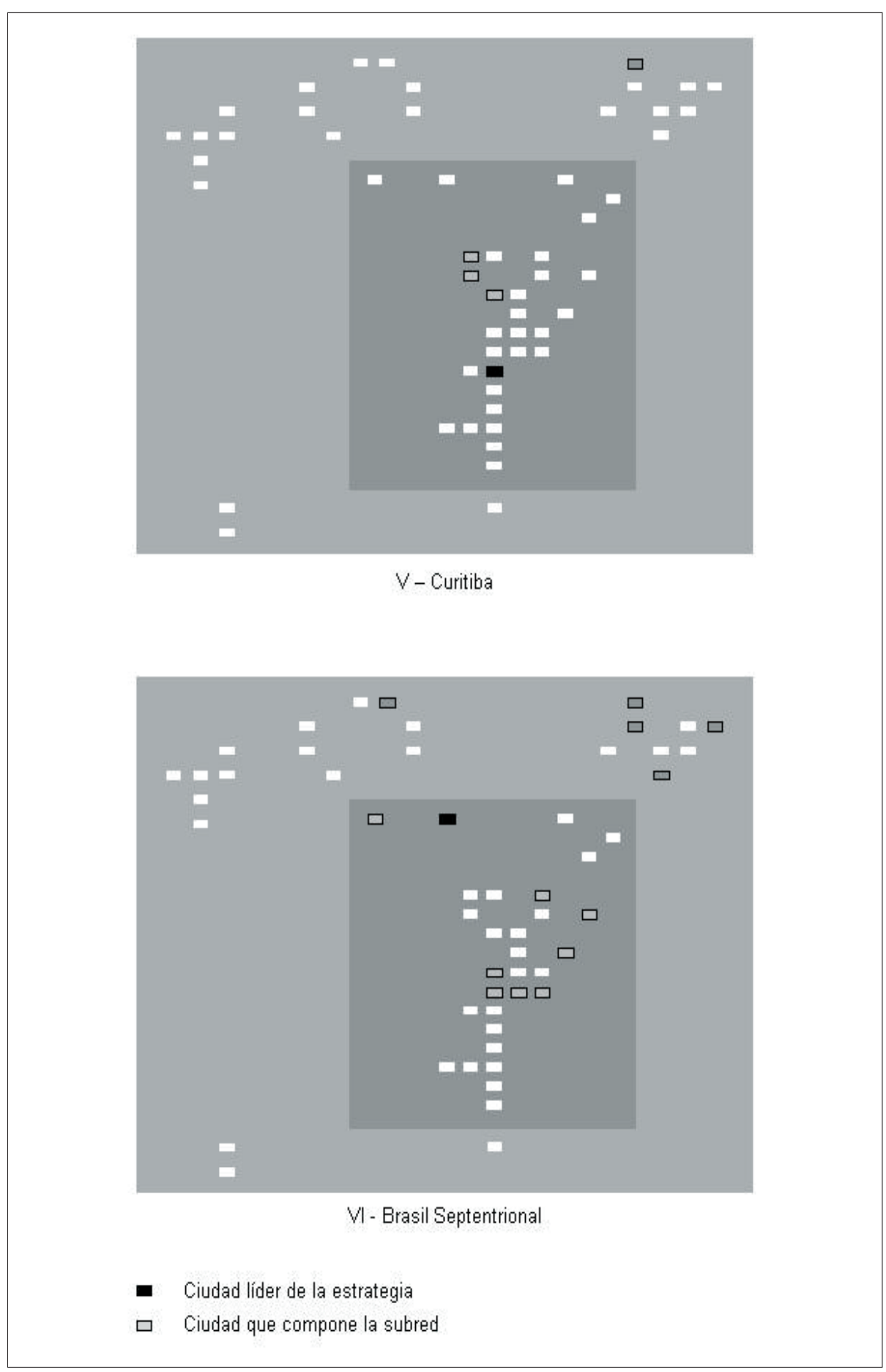

Nota: ver identificación de las ciudades en las Figuras 2 y 3.

Fuente. Elaboración propia. 
tegia VI. Las puntuaciones también revelan que esta estrategia excluye a Buenos Aires, la segunda ciudad con mayor puntuación en la estrategia de localización de los bancos en el Brasil meridional: la estrategia III.

Ocho ciudades mundiales de la muestra no tuvieron puntuaciones positivas en las subredes: Chicago, Los Ángeles y San Francisco, en Norte América; Zurich, en Europa; Taipei y Jacarta, en Asia (Pacífico); y Mumbai y Melbourne en Otras Regiones (Cuadro 1 y Figura 3). Estas ciudades definieron las estrategias a través de puntuaciones negativas, es decir, en la transposición ortogonal de las variables usadas para formar los componentes. Ellas fueron la antítesis de los objetos que alcanzaron puntuaciones positivas. La estrategia III, en particular, contó, en todas ellas, con una puntuación negativa. Como hemos visto, la estrategia III agrupa ciudades del Brasil meridional y ciudades mundiales. Entonces, las que están en referencia se encuentran totalmente distantes de ese contexto. Por otro lado, estas ciudades no contribuyeron para determinar las dos estrategias más importantes, la I y la II, las cuales presentaron la mayor varianza. Esos casos muestran que el sistema bancario que opera en Brasil prescindió de más del $30 \%$ de las ciudades mundiales de la muestra, de todas las regiones, en el período estudiado.

Aunque todas las ciudades brasileñas alcanzasen puntuaciones positivas, entre las diez ciudades con mayor producción en ventas acumuladas en el período de 1996 a 2002 -San Pablo, Río de Janeiro, Belo Horizonte, Porto Alegre, Brasilia, Salvador, Curitiba, Campinas, Vitoria y Manaos - sólo Curitiba no obtuvo ninguna puntuación negativa. Una vez más, Curitiba se comportó de manera diferente. Ciudades con elevada producción en ventas acumuladas tuvieron puntuaciones negativas en estrategias menores; por ejemplo, San Pablo y Río de Janeiro en la estrategia V; Belo Horizonte, en la IV. Mientras tanto, Vitoria, Manaos, Brasilia y Salvador tuvieron puntuaciones negativas en la más importante de las estrategias: la que conecta ciudades brasileñas al $60 \%$ de las ciudades mundiales de la muestra, la estrategia II, comprobando que aún se encuentran poco atendidas por los bancos extranjeros.

\section{Las gateway cities brasileñas}

Por un lado, ser la ciudad líder de la subred de un componente es una condición de la gateway city, de Pred, pues la puntuación elevada es un indicador de flujo intenso de informaciones bancarias. La subred de un componente representa un conjunto de ciudades que los bancos valoran empleando un mismo principio. Las ciudades líderes figuran en todas las redes de los bancos agrupados. Por ejemplo, la estrategia I reúne todas las ciudades sedes nacionales de bancos de la muestra, pero sólo San Pablo y Río de Janeiro albergan agencias de todos los bancos agrupados. Por otro lado, no siempre toda ciudad que lidera un componente será una gateway city. Tenemos que observar la ciudad líder en el contexto de la ciudad mundial, que es el elemento determinante del proceso que estamos estudiando. Además de eso, ella está ubicada en la región estudiada, como en este caso Brasil.

Las seis subredes fueron lideradas por cuatro ciudades: San Pablo, Nueva York, Curitiba y Belén. Nueva York es la segunda ciudad más importante de la muestra de ciudades mundiales, pero se encuentra lejos de Brasil. El componente III expresa la influencia de Nueva York en una subred que mezcla ciudades mundiales y ciudades brasileñas. El agrupamiento de bancos en el componente III refuerza el hecho de que Nueva York no es una gateway city: la gran mayoría son bancos brasileños liderados por un banco español. Ninguno de ellos ejerce control a través de Nueva York, pero todos están presentes en Nueva York. Belén tampoco lo será, porque todavía no es una ciudad mundial. Es una ciudad brasileña en proceso de mundialización. Los APS, que en ella operan, son controlados por la sede nacional de los bancos extranjeros agrupados, ABN-AMRO y HSBC: San Pablo y Curitiba, siendo que Curitiba no obtuvo puntuación. De esta forma, el componente VI expresa una estrategia de localización diferenciada, pero sin autonomía.

San Pablo lidera tres componentes. Dos nacionales y uno mundial. Dos de estos componentes son los más importantes: el de las ciudades brasileñas dominantes en el sistema bancario, estrategia I; y el de las ciudades mundiales, estrategia II. San Pablo es sede nacional de 12 de los 14 bancos 
Cuadro 5. Desempeño de las diez ciudades brasileñas con mayor producción en ventas en el período entre 1996 y 2002 .

\begin{tabular}{|c|c|c|c|c|c|c|}
\hline \multirow[t]{2}{*}{ Ciudad } & \multicolumn{6}{|c|}{ Estrategias } \\
\hline & I & II & III & IV & $\mathrm{V}$ & VI \\
\hline San Pablo & 2,82 & 2,38 & 1,16 & 2,83 & & 1,57 \\
\hline Río de Janeiro & 2,79 & 0,81 & & 0,62 & & \\
\hline Belo Horizonte & 2,33 & & & 1,03 & & \\
\hline Porto Alegre & 2,03 & & 0,83 & & & \\
\hline Brasilia & 1,96 & & 0,6 & & & \\
\hline Salvador & 2,04 & & & & & \\
\hline Curitiba & 1,25 & & & 0,73 & 5,53 & \\
\hline Campinas & & & & 2,68 & & \\
\hline Victoria & & & & & & 0,66 \\
\hline Manaos & & & & & & 1,84 \\
\hline
\end{tabular}

Nota: los números corresponden a la puntuación de la ciudad en la respectiva estrategia.

Fuente. Elaboración propia.

extranjeros de la muestra y de 8 de los 17 bancos nacionales. La mayor parte opera a través de redes de agencias en Brasil. Estos hechos definen San Pablo como ciudad mundial y gateway city.

Curitiba, actualmente, emerge como ciudad mundial y gateway porque, además de ser la sede nacional de dos bancos extranjeros que operan en Brasil, a través de una red de agencias, el componente V excluyó a San Pablo.

\section{El desempeño de las 10 ciudades con mayor producción en ventas}

El Cuadro 5 relaciona las diez principales ciudades brasileñas de la muestra clasificada por su producción en ventas en el período entre 1996 a 2002. Sólo San Pablo y Río de Janeiro están presentes en las dos estrategias más importantes, I y II. El desempeño de Curitiba supera, en este estudio, los de Belo Horizonte, Porto Alegre, Brasilia y Salvador, ciudades con una producción en ventas más expresiva. Aunque sea la principal ciudad administrativa brasileña y sede nacional de dos bancos brasileños con extensas redes, el desempeño de Brasilia en las relaciones bancarias transnacionales fue mediano.

Ya fue observada la falta de correlación entre producción y conectividad global de las ciudades de la muestra a través del sistema bancario (Rossi y Taylor, 2005). En aquel estudio, Manaos y
Vitoria presentaron conectividad global mediana, pero superior a la de Belén, en función de la mayor incidencia de bancos extranjeros con agencias en esas dos ciudades. En el presente estudio, que partió de la misma matriz de ciudades por bancos, Manaos y Vitoria sólo alcanzaron puntuación en el componente menor y último: el componente liderado por Belén, la vigésima ciudad en producción. Pero, mientras Manaos presenta una puntuación elevada, Vitoria tiene una puntuación en el límite. Ese resultado inesperado especificó a Manaos y Vitoria como ciudades desestimadas por los bancos de la muestra para localizar agencias con mayor poder de decisión. Además, realzó el hecho que Belén sea la sede nacional de un banco brasileño, lo que determinó su notable puntuación en el componente doméstico más importante, la estrategia I, en la que ni Manaos ni Vitoria obtuvieron puntuación. Fortaleza y Recife, respectivamente, la décima sexta y la décima primera ciudades en producción, también superaron a Manaos (décima) y a Vitoria (novena) en el primer componente. El motivo es idéntico: son sedes nacionales de bancos brasileños de la muestra. Otra ciudad que figura entre las diez mayores en producción, pero no aparece en la estrategia I, es Campinas, aunque sea vicelíder de una estrategia menor, la IV, que reúne ciudades del núcleo económico brasileño. 


\section{Conclusión}

San Pablo lidera tres flujos de informaciones bancarias. Dos de ellos, restrictos a ciudades brasileñas, son domésticos. El tercero, que incluye una ciudad de Brasil y catorce ciudades mundiales, presenta la mayor outland de las seis estrategias de localización. Además de eso, San Pablo participa de otros dos flujos transnacionales: uno involucrando ciudades del Brasil meridional, y el otro del Brasil septentrional. Este liderazgo y esta amplia envergadura hacen de San Pablo una gateway city, tal como Pred la definió, y muestra que San Pablo está ejerciendo la función de articular las economías nacional y global.

Con relación a la segunda hipótesis, el análisis de los datos mostró que la producción en ventas de las ciudades brasileñas contribuyó poco para su desempeño en el ambiente globalizado. Al revés, una ciudad ubicada en la séptima posición surgió como la probable rival de San Pablo en el papel de articular las economías nacional y global: Curitiba. Río Janeiro fue obscurecida por San Pablo en las tres estrategias en las que ambas están presentes. Campinas, Vitoria y Manaos fueron aventajadas por ciudades con producción menor. Brasilia no aparece como ciudad mundial.

El estudio mostró que el proceso de globalización contemporáneo inserta ciudades brasileñas que usualmente no son interpretadas como mundiales en la red de ciudades mundiales, evidenciando la simultánea ocurrencia de dispersión y concentración en el ambiente urbano brasileño. Además de eso, reveló que la composición de las redes articuladas por San Pablo denota selectividad: los indicios de que el proceso de globalización puede marginar ciudades importantes e incluir ciudades menores quedaron evidentes.

\section{Referencias bibliográficas}

Abril (1996-2003). As 500 maiores empresas por vendas. En Abril (Ed.), EXAME melhores e maiores. San Pablo, Brasil: Autor.

(2003a). Os 50 maiores bancos por patrimonio. En Abril (Ed.), EXAME Melhores e Maiores. San Pablo, Brasil: Autor.

(2003b). Pesquisa [versión electrónica]. En Abril (Ed.), EXAME Melhores e
Maiores. San Pablo, Brasil: Autor. Recuperado del 1 de octubre de 2003 al 30 de noviembre de 2003, de http://app.exame.abril.com. br/servicos/melhoresemaiores/ .

Burghardt, A. F. (1971). A hypothesis about gateway cities. Annals of the Association of American Geographers, 61, 2, 269-285.

Carvalho, F. J. C. (2000). New competitive strategies of foreign bank in large emerging economies: the case of Brazil. Banca nacionale del lavore quarterly review, 213, 135-169.

Chion, M. (2002). Metropolitan dimension of globalization: Lima at the end of the 20(th) century. EURE, 28, 85, 71-87.

Christaller, W. (1933). Die zentralen Orte in Süddeutschland. Jena: Gustav Fischer.

Drennan, M. P. (1992). Gateway cities: the metropolitan sources of US producers service exports. Urban Studies, 29, 2, 217-235.

Friedmann, J. (1986). The world city hypothesis. Development and change, 17, 1, 69-83.

(1995). Where we stand: a decade of world city research. En Knox, P. L. \& Taylor, P. J. (Eds.), World cities in a world-system (pp. 2147). Cambridge: Cambridge University Press.

Garza, G. (2005). Financial concentration in México City (1960-2001). EURE, 31, 92, 29-46.

Grant, R. (2001). Liberalization polices and foreign companies in Accra, Ghana. Environment and planning A, 33, 997-1014.

Grant, R. \& Nijman, J. (2002). Globalization and the corporate geography of cities in the lessdeveloped world. Annals of the Association of American Geographers, 92, 2, 320-340.

IPEA, IBGE \& UNICAMP (2001). Caracterização e tendências da rede urbana do Brasil. (Vol 1). Brasilia, DF, Brasil: IPEA.

Kowarick, L. \& Campanario, M. (1986). Sao Paulo - The price of world city status. Development and change, 17, 1, 159-174.

McKenzie, R. D. (1933). Metropolitan community. New York: Russel y Russel.

Parnreiter, C. (2005). Tendencias de desarrollo en las metrópolis latinoamericanas en la era de la globalización: los casos de Ciudad de México y Santiago de Chile. EURE, 31, 92, 5-28.

Pred, A. (1977). City Systems in advanced economies. New York: Halsted Press. 
Rossi, E. C. \& Taylor, P. J. (2005). Banking networks across Brazilian cities: interlocking cities within and beyond Brazil. Cities, 22, 5 , 381-393.

(2006). 'Gateway cities' in globalization: how banks are using Brazilian cities. Tidjschrift voor economische en social geografie, 97, 5, 513-532.

Rossi, E. C., Beaverstock, J. V. \& Taylor, P. J. (2007). Transaction links through cities: 'decision cities' and 'service cities' in outsourcing by leading brazilian firms. Geoforum, 38, 4, 628-642.

Rummel, R. J. (1970). Applied factor analysis. Evanston: Northwestern University Press.

Sassen, S.(2001). The global city. Princeton, NJ: Princeton University Press.

Sanchez, F. \& Moura, R. (2005). Model-cities: convergent strategies for their international diffusion. EURE, 31, 93, 21-34.

Taschner, S. P. \& Bogus, L. M. M. (2001). Sao Paulo, un unequal metropolis. EURE, 27, 80, 87-120.
Taylor, P. J. (2001). Specification of the world city network. Geographical analysis, 33, 2, 181-194.

(2004). World city network - A global urban analysis. London: Routledge.

Taylor, P. J. \& Walker, D. R. F. (2001). World cities: a first multivariate analysis of their services complexes. Urban Studies, 38, 1, 23-47.

Taylor, P. J., Catalano, G. \& Walker, D. R. F. (2002). Exploratory analysis of the world city network. Urban Studies, 39, 13, 2377-2394.

(2004), Multiple globalisations: regional, hierarchical and sector articulations of global business services through world cities. The Service Industries Journal, 24, 3, 63-81.

Tolosa, H. (2003). The Rio/Sao Paulo extended metropolitan region: a quest for global integration. Annals of Regional Science, 37, 3, 479-500.

Ullman, E. L. (1957). American commodity flow: a geographical interpretation of rail and water traffic based on principles of spatial interchange. Seatle: University of Washington Press. 